\title{
PROJECT OF AN APPLICATION TO FACILITATE ACCESS TO BREAST CANCER DIAGNOSIS IN CEARÁ
}

Luiz Gonzaga Porto Pinheiro1, Márcio Marcondes Vieira², Paulo H D Vasques²

${ }^{1}$ Universidade Federal do Ceará - Fortaleza (CE), Brazil.

${ }^{2}$ Centro de Oncologia Leonardo Da Vinci - Fortaleza (CE), Brazil.

Breast cancer (BC) is the malignant neoplasm most associated with mortality among women in Ceará. Its survival rate is directly related to early diagnosis. Traditionally, this diagnosis is made after the self-detection of a nodule by the patient. Mammography screening covers $43 \%$ of the target population. The diagnosis is almost exclusively made in advanced stages, leading to high mortality (3735 deaths from 2013 to 2018). Raising awareness of the population is crucial to increase mammography coverage. The development of an application to refer suspected cases to a specialized care unit may increase the early diagnosis of the disease and improve its prognosis. The project consists of developing an application that directs women to get mammographies, increasing the care coverage, informing them about risk factors, helping them find reference centers, collecting data, and generating reports and predictions. 